\title{
TEM analysis of Si thin films prepared by diode laser induced solid phase epitaxy at high temperatures
}

\author{
T. Schmidt ${ }^{\bowtie, a}$, J. L. Lábár ${ }^{\mathrm{b}}$, F. Falk ${ }^{\mathrm{a}}$ \\ a Institute of Photonic Technology, Albert-Einstein-Straße 9, 07743 Jena, Germany \\ b Institute for Technical Physics and Material Science; Research Centre for Natural \\ Sciences of the Hungarian Academy of Sciences, Konkoly Thege M. u. 29-33, 1121 \\ Budapest, Hungary \\ $\square \quad$ Author to whom any correspondence should be addressed: \\ thomas.schmidt@ipht-jena.de \\ phone: +493641206401 \\ fax: +493641206499
}

\begin{abstract}
The solid phase crystallization of amorphous silicon thin films deposited on $<111>$-oriented wafers at high temperatures was investigated. The films were heated up by diode laser irradiation for some milliseconds to seconds. Time resolved reflectivity measurements together with numerical temperature calculations showed that temperatures above $1000^{\circ} \mathrm{C}$ were reached before significant crystallization took place. By comparing two different laser intensities it is shown that random nucleation and growth dominate solid phase epitaxy if high temperatures are reached faster. The interface between epitaxially grown and randomly crystallized material is very rough which can be attributed to contaminations found at the substrate-film interface.
\end{abstract}

\section{Keywords}

Solid phase epitaxy; Random nucleation and growth; IR laser; Silicon; Time resolved reflectivity measurements; TEM analysis

\section{Introduction}

Solid phase epitaxy (SPE) of thin amorphous silicon films on crystalline or polycrystalline substrates is a common technology in transistor fabrication on monocrystalline substrates $[1,2,3]$. It was also shown, that SPE is suitable for producing crystalline silicon thin film solar cells based on a polycrystalline seed layer prepared by aluminum-induced crystallization $[4,5]$ or by diode laser crystallization [6].

Many investigations concerning the crystallization kinetics were carried out with furnace or hot plate annealing at relatively low temperatures around $600^{\circ} \mathrm{C}[7,8,9,10]$. In this case the heating-up time is small as compared to the time needed for crystallization of amorphous silicon. Thus the process can be evaluated in terms of a 
constant temperature $[7,11]$. Furthermore, the nucleation rates at this temperature are small enough to not disturb an epitaxial regrowth significantly, so that it can be neglected in most cases.

Some experiments based on halogen lamp annealing [12] and argon or dye laser heating were reported [11], dealing with temperatures up to $1000^{\circ} \mathrm{C}$ and above. In this case the temperature rise during crystallization has to be taken into account since the growth velocity and nucleation rate increase rapidly with temperature [13]. Moreover, the ratio between growth velocity and nucleation determines which part of the amorphous volume is crystallized by SPE and which one by random nucleation and growth (RNG).

In this work solid phase epitaxy of amorphous silicon layers on monocrystalline substrates is evaluated by TEM-investigations. In view of a later usage in seed layer based absorber thickening for crystalline thin film solar cells (see [6]) a diode laser is used for heating up and crystallizing the films within microseconds to seconds. To evaluate the worst case for an epitaxial thickening wafers with $\langle 111\rangle$-orientation were used as a substrate. This orientation is known to grow most defective and slowest in SPE $[14,15]$.

\section{Material and methods}

$<111>$-oriented, one-side polished n-type wafers with a dopant concentration of $8 \times 10^{-18} 1 / \mathrm{cm}^{3}$ were used as substrates. The wafers were cut into 1 "x 1 " square samples to fit in the laser setup. A standard photoresist on the polished side was used to protect the surface during cutting. The resist was then removed in piranha solution $\left(\mathrm{H}_{2} \mathrm{SO}_{2}: \mathrm{H}_{2} \mathrm{O}_{2}=4: 1,15 \mathrm{~min}\right)$ and a standard RCA clean [16] followed. After the cleaning procedure $550 \mathrm{~nm}$ of amorphous silicon were deposited by electron beam evaporation at $250^{\circ} \mathrm{C}$. During deposition the working pressure was $1 \times 10^{-7} \mathrm{mbar}$. The deposition rate was $270 \mathrm{~nm} / \mathrm{min}$.

For laser treatment of the samples a diode laser $(\lambda=808 \mathrm{~nm})$ was used with a well homogenized $1 \times 1 \mathrm{~mm}^{2}$ top hat profile spot. For diagnostics a helium neon probe laser $(\lambda=633 \mathrm{~nm})$ is focused at the center of the diode laser spot. During irradiation the reflectivity of the sample is recorded (time resolved reflectivity, TRR). The focus of the helium neon probe laser has a circular Gaussian shape with a diameter of $150 \mu \mathrm{m}$, which is small enough to observe an area of nearly constant temperature on the irradiated sample. More details concerning the probe laser setup or the beam shape of the lasers can be found in [17], where, in contrast to this work, the reflectivity was measured from the backside of the sample.

TEM cross sectional lamellae were prepared from the irradiated spots by mechanical cutting, embedding in a Ti-disk and polishing down to $50 \mu \mathrm{m}$. Then $\mathrm{Ar}^{+}$-ion milling at 7 $\mathrm{kV}$ was applied till perforation. Finally the surface of the lamellae was cleaned first with $3 \mathrm{kV}$ then with $300 \mathrm{eV} \mathrm{Ar}^{+}$-ions. The cross sections were examined in a JEOL3010 HRTEM equipped with a GATAN GIF Tridiem imaging filter. The TEM images were recorded at $300 \mathrm{kV}$ from [110] direction of the monocrystalline Si substrate.

Since it is very hard to measure the spatially varying temperature evolution over time during the irradiation, we used finite elements numerical calculations to determine the temperature profile evolution. The used equations and assumptions are described in 
[17], where a film stack on a transparent glass substrate was treated. Differing from the latter, in this work an absorbing Si wafer is used as a substrate. Since the heat conduction lengths of crystalline silicon reaches the centimeter range within the considered times, the heat loss due to the contact between wafer and substrate holder has to be taken into account. This is done by considering a heat flux $h\left(T-T_{\mathrm{R}}\right)=$ $\vec{n}(\kappa \nabla T)$ at the contact area defining a Neumann type boundary condition. $\kappa$ is the thermal conductivity, $\vec{n}$ is the normal vector, $T_{\mathrm{R}}$ is the ambient temperature, and $h$ is the heat transfer coefficient, which was chosen carefully, so that the calculated times of surface melting coincided with the measured ones. Concerning the thin film on top of the wafer the phase change between amorphous and crystalline silicon influences mainly the reflectivity of the sample. The thermal contribution of the thin film is negligible [18]. The optical properties of the film were assumed as a mixture between a$\mathrm{Si}$ and c-Si whereas the time dependent crystalline part was taken roughly from the measured TRR curves. With this the interference dependent reflectivity $R$ was calculated during simulation using temperature dependent optical parameters from [19] (a-Si) and [20] (c-Si). The equation for the depth dependent intensity distribution $\frac{\partial I}{\partial z}=-\alpha(z) I$ was solved numerically for each time step, with the absorption coefficient $\alpha(z)$ from $[19,20]$. Finally the absorbed intensity is calculated according to (1$R) I_{0}(x, y) I(z)$, where $I_{0}(x, y)$ is the spatial profile of the incident laser beam.

\section{Results and discussion}

Two sets of laser parameters were used for crystallization of the film: in set 1 the spots were irradiated with $12.5 \mathrm{~kW} / \mathrm{cm}^{2}$ for only $80 \mathrm{~ms}$ and in set 2 with $7.6 \mathrm{~kW} / \mathrm{cm}^{2}$ for 2500 ms. In the following the spots irradiated with the two different sets are called spot 1 and 2 , respectively. Figure 1 shows the TRR signal together with the calculated temperature evolutions of the two spots irradiated with different laser intensities. At the beginning the reflectivity rises due to the temperature increase and the related increase in the refractive index of silicon. After a certain while the reflectivity drops which is a clear indicator for the solid phase change from amorphous to crystalline silicon and the decrease in refractive index which comes along with it. This continuous drop also suggests that there is no flat epitaxy front reaching the surface since this would lead to a periodical decrease and increase in the reflectivity due to interference effects [10, 21]. The related faster rise in the calculated temperature curves is due to the decrease in reflectivity for the heating laser, which leads to an increase in absorbed power.

Eventually the laser pulse heats up the crystalline silicon until the irradiation stops and the material cools down. When comparing the two experiments one can see that the solid phase change takes place in almost the same temperature range around $1050^{\circ} \mathrm{C}$ despite of the two different time evolutions. Since in spot 2 this range is reached later and is passed much more slowly than in spot 1 , the corresponding solid phase change needs $400 \mathrm{~ms}$ instead of $40 \mathrm{~ms}$. 
Materials Letters 122 (2014) 37-40

http://dx.doi.org/10.1016/j.matlet.2014.02.006
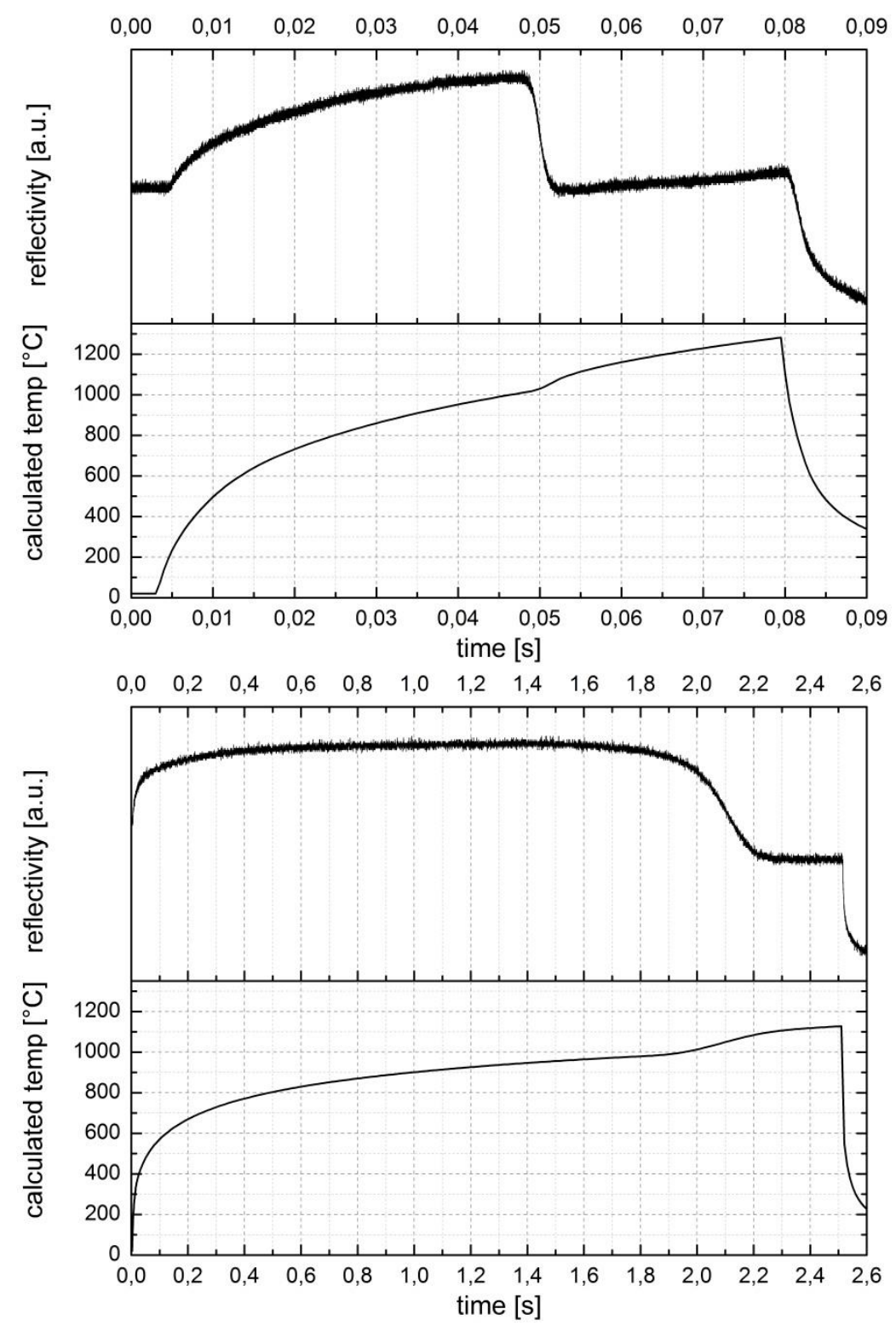

Figure 1: Measured reflectivity and calculated temperature for spots 1 (top) and 2 (bottom) with different laser intensities and irradiation times (spot 1: $80 \mathrm{~ms}$ at $12.5 \mathrm{~kW} / \mathrm{cm}^{2}$, spot 2: $2500 \mathrm{~ms}$ at $7.6 \mathrm{~kW} / \mathrm{cm}^{2}$ ). Take note of the different time scales.

Figure 2 shows the TEM overview images of cross sections prepared at the center of the two aforementioned spots. It is clearly visible that the whole film was crystallized. The dark colored regions in the film have almost the same shading as the substrate which indicates the same crystallographic orientation and suggests that these regions were grown epitaxially. The orientation of the layer was also confirmed by selected area electron diffraction (SAED) patterns. In contrast to this the brighter regions above indicate grain orientations different from the substrate and were therefore crystallized by random nucleation and growth. Nevertheless all regions show strong varying shades of grey which indicates a high density of defects even in the epitaxially grown part, which is not surprising since the substrate was $<111>$-oriented. 

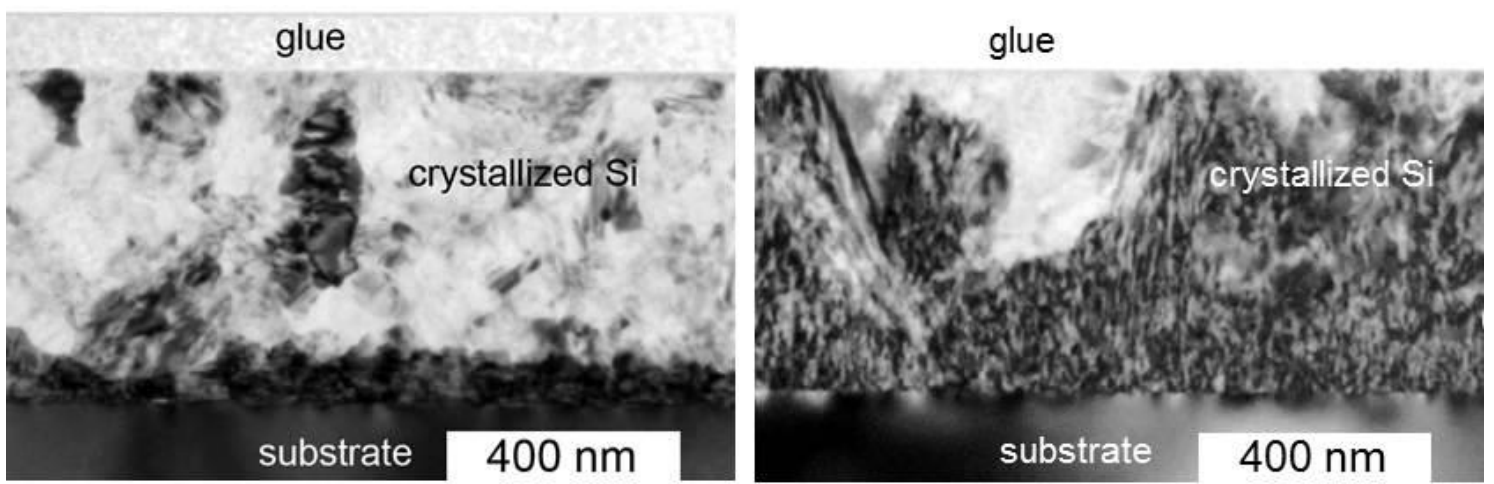

Figure 2: TEM overview images for spot 1 (left) and 2 (right). The amorphous silicon was crystallized completely. The substrate-near part was grown epitaxially and is full of defects, whereas top part crystallized spontaneously.

Figure 3 shows a TEM image and corresponding SAED patterns taken from spot 2. By comparing Figure $3 a$ ) and b) one can see that the dark regions have in fact the same crystallographic orientation as the substrate but are full of twins. In contrast to this, diffraction patterns of the bright regions show either polycrystalline material (c) or slightly misoriented grains (d).
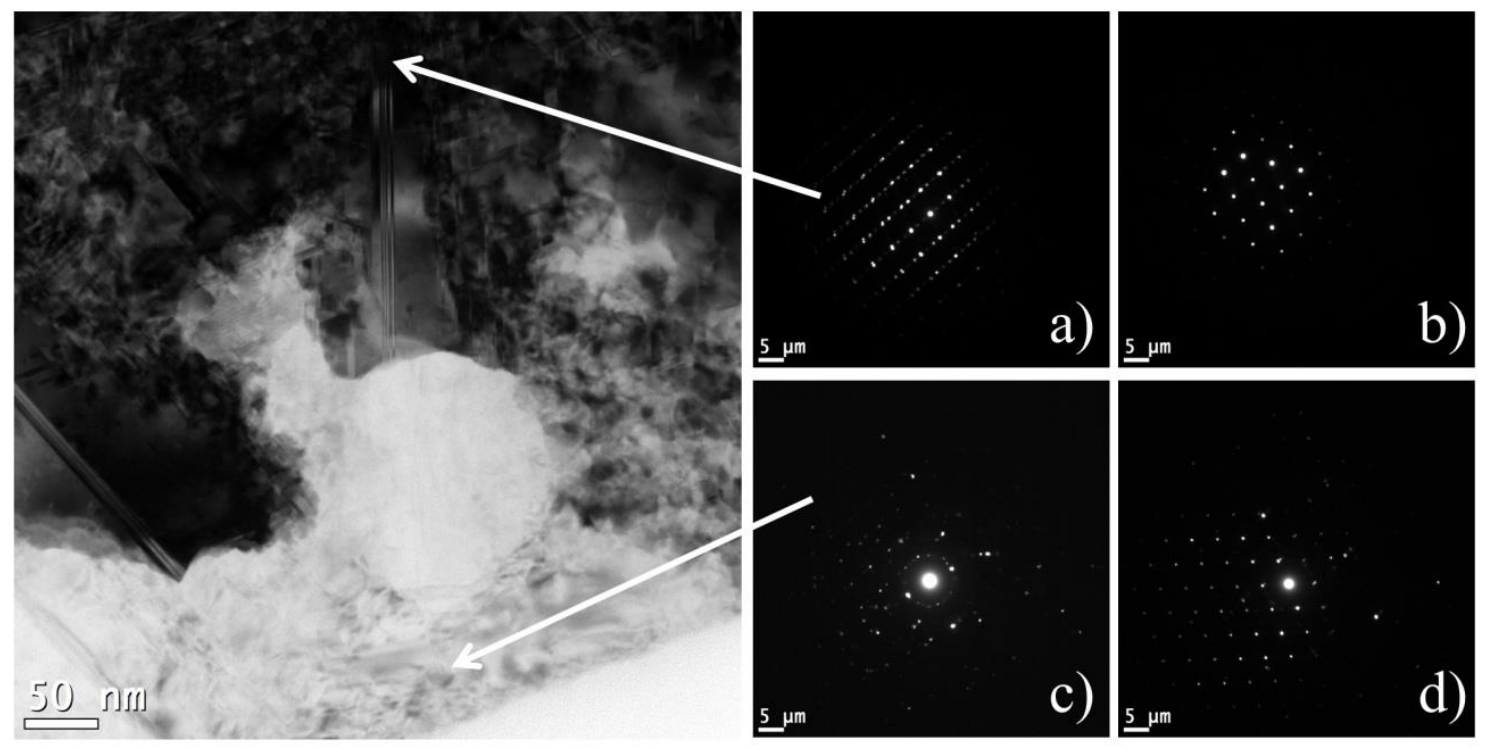

Figure 3: TEM image and diffraction pattern of selected film positions in spot 2. a) dark region, b) substrate, c) bright region - polycrystalline, d) bright region - misoriented. Images a) and c) are related to the left TEM image whereas $b$ ) and d) where taken from another image.

By regarding the SPE grown material within spot 1 and 2 in Figure 2 one can observe, that in spot 1 only the first $100 \mathrm{~nm}$ tended to crystallize epitaxially whereas in spot 2 this range is much wider and even reaches the surface of the sample in some regions. Concerning the growth velocity and the nucleation rate this means that in this temperature range the nucleation rate rises faster with the temperature than the growth velocity does (within spot 1 the film was heated up much more rapidly than in spot 2).

Moreover, it is obvious that the interface between the SPE and RNG regions is very rough which was already indicated by the TRR measurements. The reason for that is 
unclear so far, but may be related to interface contaminations which can retard epitaxy $[22,23]$. Figure 4 shows a high resolution image of the interface between the substrate and the epitaxially grown layer. It is clearly visible that the contrast is smeared out over 1 to 4 atomic layers. This could be attributed to interface imperfections like contaminations with foreign atoms. The varying thickness of the contamination layer leads to spatially different retardation times of the SPE and therefore to the rough interface between SPE and RNG regions.

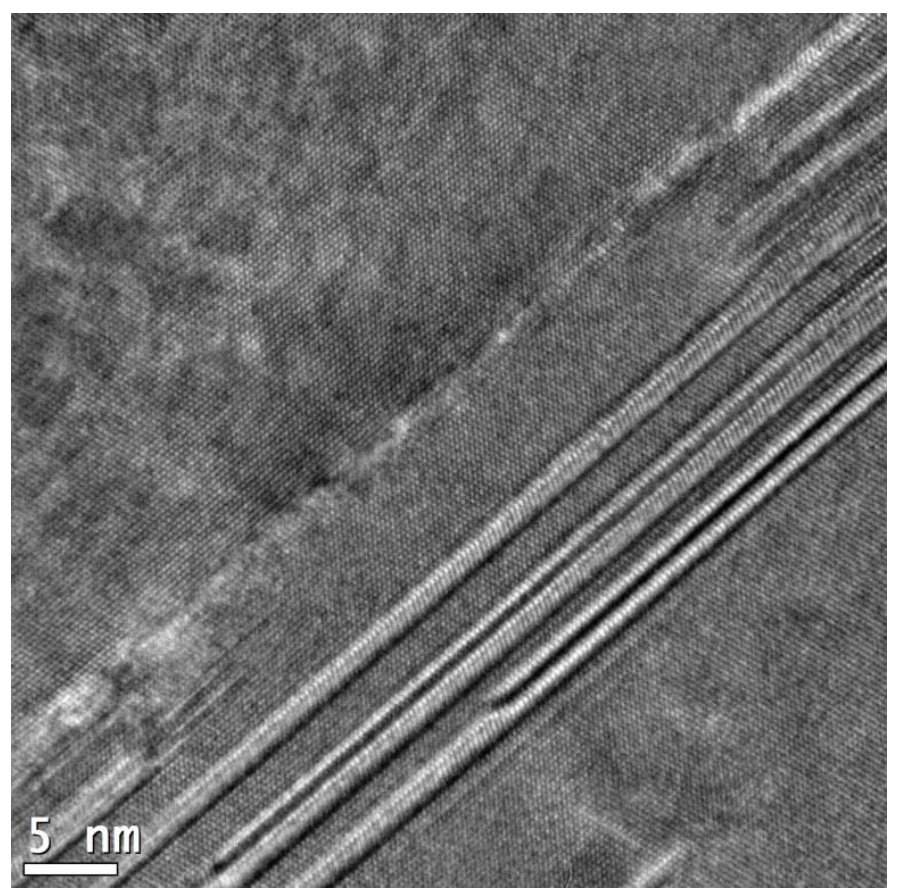

Figure 4: High resolution TEM image from the interface between substrate and epitaxially grown layer. The smeared out contrast next to the interface is caused by atoms not lying in the $\langle 110\rangle$ direction, which was chosen for preparation.

\section{Conclusions}

In this work high temperature solid phase epitaxy of evaporated amorphous silicon on $<111>$-oriented wafers was investigated. The thin films were heated up with a diode laser for milliseconds up to seconds and the solid phase change was observed by TRR measurements. Numerical temperature simulations were done revealing that the phase change takes place around $1050^{\circ} \mathrm{C}$ whereas the time needed for completion of crystallization depends on the heating rate and therefore on the laser intensity. Cross section TEM investigations show that the irradiated areas crystallized completely, but are full of defects. Therefore the films showed epitaxially grown regions near to the substrate and regions crystallized by RNG. The SPE grown regions were larger for spots irradiated with lower intensities. This leads to the conclusion that the nucleation rate rises faster with the temperature than the growth velocity does. The interfaces between the SPE and RNG regions were very rough which could be attributed to imperfections visible at the substrate-film interface. 
Materials Letters 122 (2014) 37-40

http://dx.doi.org/10.1016/j.matlet.2014.02.006

\section{References}

[1] O. Skibitzki, Y. Yamamoto, M. A. Schubert, B. Tillack. Solid-phase epitaxy of undoped amorphous silicon by in-situ postannealing. Thin Solid Films 2012; 520: $3271-3275$.

[2] M. Sasaki, T. Katoh, H. Onoda, N. Hirashita Lateral solid-phase epitaxy of Si over $\mathrm{SiO} 2$ patterns and its application to silicon-on-insulator transistors. Appl Phys Lett 1986; 49: 397-399.

[3] K. Ishii, Y. Hayashi, T. Sekigawa. Experimental fabrication of XMOS transistors using lateral solid-phase epitaxy of CVD silicon films. Jpn J Appl Phys, Part 2, Lett 1990; 29: L521-L523.

[4] P. Prathap, O. Tuzun, D. Madi, A. Slaoui. Thin film silicon solar cells by AIC on foreign substrates. Sol Energy Mater Sol Cells 2011; 95: S44-S52.

[5] A. Aberle. Progress with polycrystalline silicon thin-film solar cells on glass at UNSW. J Cryst Growth 2006; 287: 386-390.

[6] G. Andrä, J. Bergmann, A. Gawlik, J. Plentz, I. Höger, T. Schmidt, F. Falk. Thin film solar cells based on diode laser crystallized polycrystalline silicon. 26th Eur Photovolt Sol Energy Conf Exhib 2011: 2803-2806.

[7] I. Hoeger, A. Gawlik, G. Andrae, F. Falk. Thickening of thin laser crystallized silicon films by solid phase epitaxy for photovoltaic applications. J Cryst Growth 2013; 364: 164-168.

[8] X. Maeder, C. Niederberger, S. Christiansen, A. Bochmann, G. Andrä, A. Gawlik, F. Falk, J. Michler. Microstructure and lattice bending in polycrystalline laser-crystallized silicon thin films for photovoltaic applications. Thin Solid Films 2010; 519: 58-63.

[9] N. G. Rudawski, K. S. Jones, S. Morarka, M. E. Law, R. G. Elliman. Stressed multidirectional solid-phase epitaxial growth of Si. J Appl Phys 2009; 105: 81101/1- 81101/20.

[10] M. Bauer, M. Oehme, M. Sauter, G. Eifler, E. Kasper. Time resolved reflectivity measurements of silicon solid phase epitaxial regrowth. Thin Solid Films 2000; 364: 228-232.

[11] G. Olson, J. Roth. Kinetics of solid phase crystallization in amorphous silicon. Mat Sci Rep 1988; 3: 1-78.

[12] O. Tuezuen, A. Slaoui, S. Roques, A. Focsa, F. Jomard, D. Ballutaud. Solid phase epitaxy on $\mathrm{N}$-type polysilicon films formed by aluminium induced crystallization of amorphous silicon. Thin Solid Films 2009; 517: 6358-6363.

[13] U. Köster. Crystallization of amorphous silicon films. Phys Status Solidi A 1987; 48: 313-321.

[14] A. Zotov, V. Korobtsov. Present status of solid phase epitaxy of vacuumdeposited silicon. J Cryst Growth 1989; 98: 519-530.

[15] L. Csepregi, E. Kennedy, J. Mayer, T. Sigmon. Substrate-orientation dependence of epitaxial regrowth rate from Si-implanted amorphous Si. J Appl Phys 1978; 49: 3906-3911. 
[16] W. Kern. The evolution of silicon wafer cleaning technology. J Electrochem Soc 1990; 137: 1887-1892.

[17] T. Schmidt, A. Gawlik, H. Schneidewind, A. Ihring, G. Andrä, F. Falk. Experimental setup for investigating silicon solid phase crystallization at high temperatures. Opt Express 2013; 21: 16296-16304.

[18] T. Schmidt, I. Hoeger, A. Gawlik, G. Andrä, F. Falk. Solid phase epitaxy of silicon thin films by diode laser irradiation for photovoltaic applications. Thin Solid Films 2012; 520: 7087-7092.

[19] J. Bergmann, M. Heusinger, G. Andrä, F. Falk. Temperature dependent optical properties of amorphous silicon for diode laser crystallization. Opt. Express 2012; 20: A856-A863.

[20] Y. Sun, X. Zhang, C. Grigoropoulos. Spectral optical functions of silicon in the range of 1.13-4.96 eV at elevated temperatures. Int J Heat Mass Transfer 1997; 40: $1591-1600$.

[21] J. Roth, G. Olson, D. Jacobson, J. Poate. Kinetics of solid phase epitaxy in thick amorphous Si layers formed by MeV ion-implantation. Appl Phys Lett 1990; 57: 1340-1342.

[22] M. von Allmen, S. Lau, J. Mayer, W. Tseng. Solidstate epitaxial growth of deposited si films. Appl Phys Lett 1979; 35: 280-282.

[23] L. Hung, S. Lau, M. von Allmen, J. Mayer, B. Ullrich, J. Baker, P. Williams, W. Tseng. Epitaxial growth of Si deposited on (100) Si. Appl Phys Lett 1980; 37: 909-911. 